\title{
SVM-Enhanced Filtering Model for Limit Order Book Dynamics
}

\author{
Hayato Kijima $^{\dagger}$, Hideyuki Takada*, Takayuki Tomiya ${ }^{\dagger \dagger}$ \\ Department of Information Science, Toho University \\ Miyama 2-2-1, Funabashi, Chiba 274-8510, Japan \\ † E-mail: 5512032k@nc.toho-u.ac.jp \\ * E-mail: hideyuki.takada@is.sci.toho-u.ac.jp \\ †† E-mail: 5512072t@nc.toho-u.ac.jp
}

\begin{abstract}
We propose a stochastic filtering model for Limit Order Book (LOB) where the signal process obeys the stochastic differential equation (SDE) whose trend function depend on the history of the LOB in such a way as to reflect the predicted direction of the mid price movement via the Support Vector Machine (SVM). Stochastic dynamics of the LOB is modeled with a queuing system where incoming orders and cancellations of existing orders arrive according to conditionally independent Poisson processes. Finally, numerical example with Nikkei 225 futures are presented.
\end{abstract}

\section{Introduction}

In this study, stochastic dynamics of the Limit Order Book (LOB) is modeled with a queuing system where incoming orders and cancellations of existing orders arrive according to independent Poisson processes as proposed by Cont et al. [3]. The main contribution of the paper could be introduction of latent factors into queuing system to represent time varying intensities. In general, market participants post or cancel their orders based on the knowledge of historical order flows and therefore order arrival rate will change in time. In order to visualize the latent factors, we assume that the latent factors are unobservable for market participants and try to estimate the distribution of them via stochastic filtering. In this framework, market participants update their belief about distribution of the latent factors by use of observable data, i.e., the history of the LOB. This enable one to estimate the degree of market activities and then the arrival rates of future market orders would be evaluated.

We further assume that stochastic dynamics of the latent factors (signal process) depend on the history of the LOB. More precisely, the latent factors are governed by the stochastic differential equation (SDE) whose trend function explicitly depend on the trajectory of the LOB in such a way as to reflect the predicted direction of the mid price move via the Support Vector Machine. This enable one to build realistic transitions of the latent factors.

This paper is organized as follows. Section 2 introduces our model and states some terminology to describe the stochastic dynamics of the LOB. Most of Section 2 is devoted to the appropriate modeling of the arrival intensities of the limit/market orders as a function of the latent factors. In section 3, we derive the normalized filter. Section 4 discuss the SDE of the latent factor and the numerical algorithm for solving filter equation is described in Section 5. Section 6 provides the numerical example for high frequency data of Nikkei 225 Futures.

\section{Model Setting}

\subsection{State of the Limit Order Book (LOB)}

We formulate the stochastic dynamics of the LOB in the context of a high-dimensional queuing system as first introduced in Cont et al. [3]. In contrast to this paper, we do not impose the restriction that all incoming and outgoing orders are of size one.

It is assumed that limit orders and market orders can be placed on a fixed price grid $\mathcal{P}=\{1,2, \cdots, n\}$ representing multiples of a price tick. Here we assume that the tick size of the LOB is denoted by $\delta$.

The upper boundary $n$ is chosen large enough so that it is highly unlikely that orders at prices higher than $n$ will be placed within the time frame of our analysis $^{1}$. State of the LOB at time $t$ is described by the continuous time $\mathbb{R}^{n}$-valued stochastic process $Z(t)=\left(Z_{1}(t), \cdots, Z_{n}(t)\right)$, where the $p$-th element $Z_{p}(t)$ denotes the time $t$ order size waiting for the future market order to be matched. If $Z_{p}(t)>0$ then $Z_{p}(t)$ represents the size of limit sell orders and if $Z_{p}(t)<0$ then $Z_{p}(t)$ represents the size of limit buy orders at time $t$ respectively. (See Fig. 1.)

The best-ask price at time $t$ is then defined by $P_{\text {sell }}(t)=\inf \left\{p \in \mathcal{P} \mid Z_{p}(t)>0\right\} \wedge(n+1)$ and similarly the best-bid price is defined by $P_{\text {buy }}(t)=$

\footnotetext{
${ }^{1}$ As described in Cont et al. [3], since the model is intended to be used on the time scale of days, this finite boundary assumption is reasonable.
} 


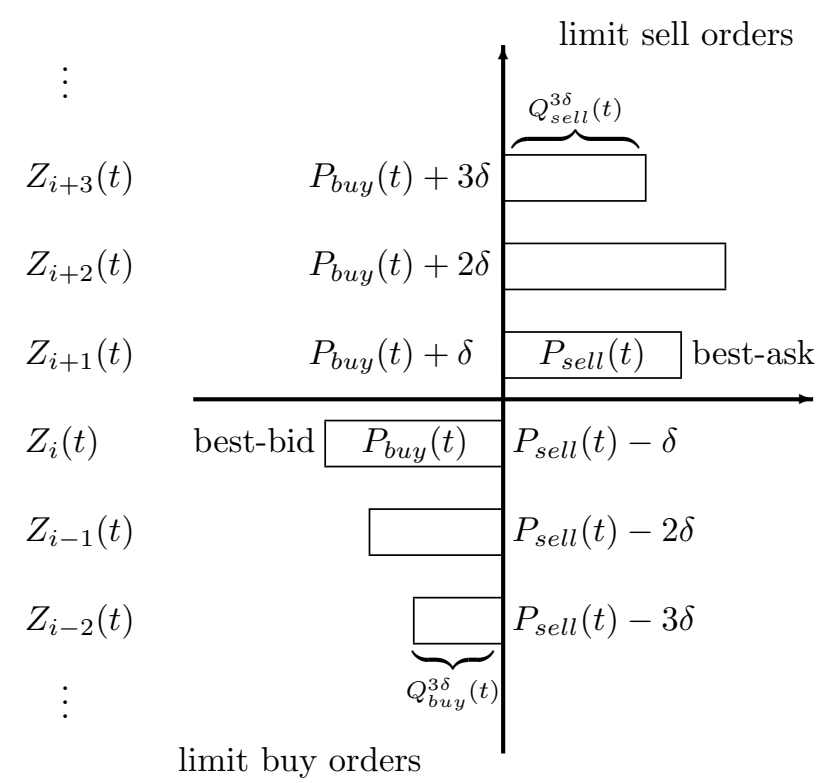

Fig. 1: The shape of the Limit Order Book at time $t$

$\sup \left\{p \in \mathcal{P} \mid Z_{p}(t)<0\right\} \vee 0$. Furthermore, we define the number of outstanding sell orders at a distance $k$ (equivalently $k \cdot \delta$ in price) from the best-bid price as $Q_{\text {sell }}^{k \delta}(t)=Z_{P_{b u y}(t)+k \delta}(t)>0$. Thus the quantity $Q_{\text {sell }}^{\delta}(t)$ indicates the number of orders of best-ask at time $t$. Similarly the number of outstanding buy orders at a distance $k$ from the best-ask price is defined as $Q_{\text {buy }}^{k \delta}(t)=Z_{P_{\text {sell }}(t)-k \delta}(t)<0$.

\subsection{Stochastic Dynamics of the Order Book}

Uncertainty in our model is modeled by a probability space $(\Omega, \mathcal{F}, \mathbb{P})$ equipped with a filtration $\left(\mathcal{F}_{t}\right)_{t \geq 0}$. We slightly extend the model proposed by Cont et al. [3] by introducing the size of limit/market orders and then the stochastic dynamics of the order book is described as follows. ${ }^{2}$ In what follows, define the set of orders $\mathcal{O}:=$ \{limit sell order, cancellation of limit sell order, limit buy order, cancellation of limit buy order, market sell order, market buy order $\}$ and these elements are labeled by the integers $\{1,2,3,4,5,6\}$. For example, order of type $3 \in \mathcal{O}$ means arrival of limit buy order.

One sees that a limit sell order, i.e., order of type $1 \in \mathcal{O}$, with $j$ units at price level $p>P_{b u y}(t)$ increases the quantity at price $p$ as $Z_{p}(t+\Delta t)=Z_{p}(t)+j$. Similarly a cancellation of an outstanding limit sell order, i.e., order of type $2 \in \mathcal{O}$, of $j$ units at price level $p>P_{\text {buy }}(t)$ decreases the quantity at price $p$ as $Z_{p}(t+\Delta t)=Z_{p}(t)-j$. The other type of orders would be described similarly so the time evolution of the LOB is driven by the incoming flow of 6 type of orders and each of them can then be represented as a counting

\footnotetext{
${ }^{2}$ In this paper, we take $\Delta t=20$ milliseconds.
}

process with mark, where the mark records the size of orders.

We assume that the arrival rate of orders described above are modulated by some latent factors. Let $X(t)=\left(X_{1}(t), X_{2}(t)\right)$, representing the potential demands for selling and buying, be a 2-dimensional unobservable state process (signal process) governed by the next SDEs

$d X_{i}(t)=a_{i}\left(t, X_{i}(t), Z_{[0, t]}\right) d t+b_{i}\left(t, X_{i}(t), Z_{[0, t]}\right) d B_{i}(t)$,

where the functions $a_{i}, b_{i}(i=1,2)$ satisfy the conditions of the linear growth and Lipschitz continuity to ensure the existence and uniqueness of strong solutions. Here, $Z_{[0, t]}$ denotes the trajectory of the process $Z$ up to time $t$, and $B_{1}(t)$ and $B_{2}(t)$ are $\mathbb{P}$-Brownian motion satisfying $d B_{1}(t) d B_{2}(t)=\rho d t, \rho \in[-1,+1]$. One can translate the role of $X(t)=\left(X_{1}(t), X_{2}(t)\right)$ as follows; process $X_{1}(t)\left(X_{2}(t)\right)$ represents selling (buying) tendency, i.e., increasing of $X_{1}(t)\left(X_{2}(t)\right)$ would mean overheating of sell (buy) orders and cancellation of the sell (buy) orders are relatively low ${ }^{3}$. Here we postulate the next model assumption.

Assumption 2.1. For given state $X(t)$, the events such that arrivals and cancellations of the limit/market orders are modeled with conditionally independent exponentially distributed inter arrival times.

\subsubsection{Arrivals of the Limit Sell Orders}

We assume that the arrival rate of limit sell order (i.e., order of type $1 \in \mathcal{O}$ ) of size $j$ to the price $p$, where $p>P_{\text {buy }}(t)$, at time $t$ is given by

$$
\begin{aligned}
& \lambda_{1}(j, p \mid X(t)) \\
& =\bar{\lambda}_{1}\left(p-P_{\text {buy }}(t), X_{1}(t), X_{2}(t)\right) \times J_{1}\left(j \mid X_{1}(t), X_{2}(t)\right),
\end{aligned}
$$

where the function $\bar{\lambda}_{1}: \mathbb{Z}_{+} \times \mathbb{R}^{2} \rightarrow \mathbb{R}_{+}$represents the arrival rate (arrival intensity) of limit sell order and $J_{1}\left(\cdot \mid x_{1}, x_{2}\right): \mathbb{Z}_{+} \rightarrow \mathbb{R}_{+}$represents the conditional probability that the incoming limit sell order has $j$ units given that the limit sell order is posted to the price level $p$.

According to empirically observed facts presented in Zovko and Farmer [9] and Bouchaud et al. [1], order arrival rates depend on the distance to the bid/ask in such a way that most orders are placed close to the current price. In addition, frequency of incoming limit sell orders would get higher within selling pressure so $\bar{\lambda}_{1}$ should be the increasing function with respect to $X_{1}(t)$. Therefore we assume

$$
\bar{\lambda}_{1}\left(\ell, x_{1}, x_{2}\right)=\frac{\exp \left(\beta x_{1}\right)}{(\ell / \delta)^{\alpha}}, \alpha, \beta>0 .
$$

In general, limit orders tend to arrive with the minimum unit or at most several fold, and huge amount of limit

\footnotetext{
${ }^{3}$ If both increases, the market is active while both decreases, the market is quiet.
} 
orders rarely come with one shot ${ }^{4}$. Therefore, $J_{1}(j)$ should be a monotonically decreasing function with respect to $j$ for fixed $X(t)$. We assume $J_{1}(j)=g(1-$ $g)^{j-1}, j=1,2, \cdots, \infty$ with some parameter $g$, which will be defined as a function of $\left(X_{1}(t), X_{2}(t)\right)$. When the net selling tendency gets large, that is, $X_{1}(t)-X_{2}(t)$ gets large, it would be appear that the amount of the limit sell order would increase. In order to determine whether the function $g$ is increasing or decreasing function with respect to $X_{1}(t)-X_{2}(t)$, let us consult the Figure 2. For example, when $g=0.1$, the shape of the function $j \mapsto J_{1}(j)=g(1-g)^{j-1}$ is shown by the solid line and $g=0.04$ case is plotted by the dashed line. When the net selling tendency $X_{1}(t)-X_{2}(t)$ is rela-

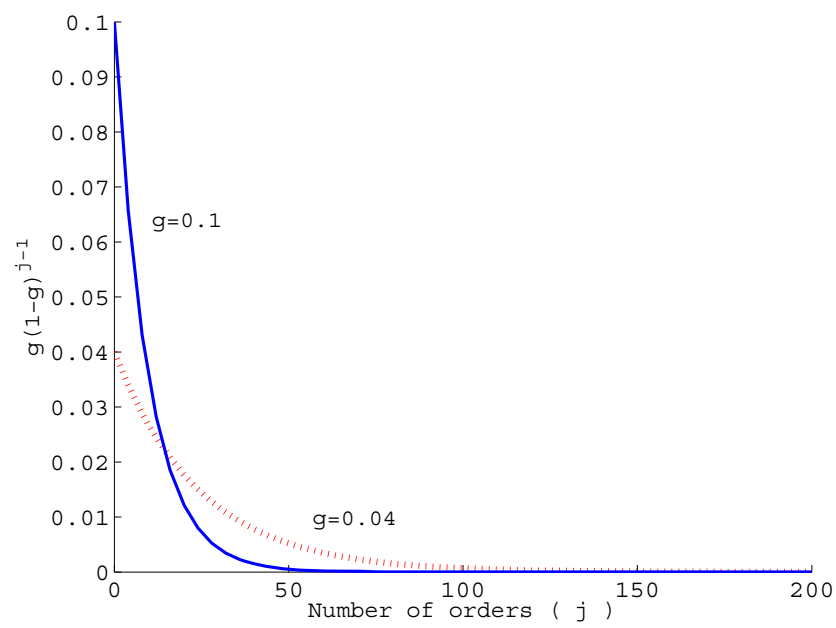

Fig. 2: The shape of the function $j \mapsto J_{1}(j)=g(1-$ $g))^{j-1}$ for $g=0.04$ and 0.1 .

tively large, the amount of the limit sell orders would increase and then such a situation corresponds to the dashed line in Figure 2 rather than the solid line. Thus we can conclude that it should be better to consider that $g$ is a decreasing function of $X_{1}(t)-X_{2}(t)$. Put it all together, we assume that

$$
J_{1}\left(j \mid x_{1}, x_{2}\right)=g\left(x_{1}-x_{2}\right)\left(1-g\left(x_{1}-x_{2}\right)\right)^{j-1},
$$

where the function $g(x): \mathbb{R} \rightarrow \mathbb{R}_{+}$is decreasing with respect to $x$. In practice, we simply chose $g(x)=$ $g_{0} \exp \left(-g_{1} \cdot x\right), g_{0}, g_{1} \in \mathbb{R}_{+}$for tractability ${ }^{5}$.

\footnotetext{
${ }^{4}$ In fact, big investors tend to place their limit orders of relatively large size but round number such as 500 or 1000 , and so on. In this paper, we consider such phenomena as an irregular event and do not pay special attention to them.

${ }^{5}$ In addition, we prefer non-linear function with respect to $x$ for the sake of accentuated extraction via filtering.
}

\subsubsection{Cancellation of the Limit Sell Orders}

We assume that the limit sell orders of size $j$ at the price $p>P_{\text {buy }}(t)$ are canceled with rate

$$
\begin{aligned}
& \lambda_{2}(j, p \mid X(t)) \\
& =\bar{\lambda}_{2}\left(p-P_{b u y}(t), X_{1}(t), X_{2}(t)\right) \times J_{2}\left(j \mid X_{1}(t), X_{2}(t)\right)
\end{aligned}
$$

and $\bar{\lambda}_{2}$ is assumed to be

$$
\bar{\lambda}_{2}\left(\ell, x_{1}, x_{2}\right)=\frac{\exp \left(-\kappa x_{1}\right)}{(\ell / \delta)^{\alpha}}, \alpha>0, \kappa>0 .
$$

And the conditional probability that $j$ unit of the limit sell orders are cancelled given that the cancellation is placed to the price level $p$ is

$$
J_{2}\left(j \mid x_{1}, x_{2}\right)=\frac{h\left(x_{1}-x_{2}\right)\left(1-h\left(x_{1}-x_{2}\right)\right)^{j-1}}{\sum_{k=1}^{K} h\left(x_{1}-x_{2}\right)\left(1-h\left(x_{1}-x_{2}\right)\right)^{k-1}} .
$$

Here we suppose that the amount of the limit sell orders waiting at the price $p$ just before the cancellation is $K$ units. By the similar argument of the previous subsection, we conclude $h$ is a increasing function with respect to $X_{1}(t)-X_{2}(t)$. In practice, we take $h(x)=h_{0} \exp \left(h_{1} \cdot x\right), h_{0}, h_{1} \in \mathbb{R}_{+}$for tractability.

\subsubsection{Arrivals of the Limit Buy Orders}

We assume that the arrival rate of limit buy order (i.e., order $3 \in \mathcal{O}$ ) of size $j$ to the price $p$, where $p<$ $P_{\text {sell }}(t)$, at time $t$ is given by

$$
\begin{aligned}
& \lambda_{3}(j, p \mid X(t)) \\
& =\bar{\lambda}_{3}\left(P_{\text {sell }}(t)-p, X_{1}(t), X_{2}(t)\right) \times J_{3}\left(j \mid X_{1}(t), X_{2}(t)\right),
\end{aligned}
$$

where $\bar{\lambda}_{3}: \mathbb{Z}_{+} \times \mathbb{R}^{2} \rightarrow \mathbb{R}_{+}$represents the arrival rate of limit buy order and defined as

$$
\bar{\lambda}_{3}\left(\ell, x_{1}, x_{2}\right)=\frac{\exp \left(\beta x_{2}\right)}{(\ell / \delta)^{\alpha}}, \alpha, \beta>0 .
$$

And $J_{3}\left(\cdot \mid x_{1}, x_{2}\right): \mathbb{Z}_{+} \rightarrow \mathbb{R}_{+}$represents the conditional probability that the incoming limit buy order has $j$ unit given that the order is posted to the price level $p$. Thus we assume

$$
J_{3}\left(j \mid x_{1}, x_{2}\right)=u\left(x_{2}-x_{1}\right)\left(1-u\left(x_{2}-x_{1}\right)\right)^{j-1},
$$

with $u(x)=u_{0} \exp \left(-u_{1} \cdot x\right), u_{0}, u_{1} \in \mathbb{R}_{+}$, decreasing function of $x$.

\subsubsection{Cancellation of the Limit Buy Orders}

We assume that the limit buy orders of size $j$ at the price $p<P_{\text {sell }}(t)$ are cancelled with rate

$$
\begin{aligned}
& \lambda_{4}(j, p \mid X(t)) \\
& =\bar{\lambda}_{4}\left(P_{\text {sell }}(t)-p, X_{1}(t), X_{2}(t)\right) \times J_{4}\left(j \mid X_{1}(t), X_{2}(t)\right),
\end{aligned}
$$


where $\bar{\lambda}_{4}: \mathbb{Z}_{+} \times \mathbb{R}^{2} \rightarrow \mathbb{R}_{+}$represents the arrival rate of cancelation and defined as

$$
\bar{\lambda}_{4}\left(\ell, x_{1}, x_{2}\right)=\frac{\exp \left(-\kappa x_{2}\right)}{(\ell / \delta)^{\alpha}}, \alpha, \kappa>0 .
$$

And $J_{4}\left(\cdot \mid x_{1}, x_{2}\right): \mathbb{Z}_{+} \rightarrow \mathbb{R}_{+}$represents the conditional probability that the amount of the cancel has $j$ unit given that the cancelation is occurred to the price level $p$.

$J_{4}\left(j \mid x_{1}, x_{2}\right)=\frac{v\left(x_{2}-x_{1}\right)\left(1-v\left(x_{2}-x_{1}\right)\right)^{j-1}}{\sum_{k=1}^{K} v\left(x_{2}-x_{1}\right)\left(1-v\left(x_{2}-x_{1}\right)\right)^{k-1}}$,

with $v(x)=v_{0} \exp \left(v_{1} \cdot x\right), v_{0}, v_{1} \in \mathbb{R}_{+}$, increasing function of $x$. Here, $K$ denotes the amount of the limit sell orders just before cancellation.

\subsubsection{Execution of the Market Orders}

Since one can hardly distinguish the two events; (i) execution of the market buy orders and (ii) cancellation of the limit sell order posted in the best ask price by observing the time series of the LOB, we assume the following. As we defined in Section 2.1, the number of the best-ask orders denoted by $Q_{\text {sell }}^{\delta}(t)$ and the number of best-bid orders denoted by $Q_{b u y}^{\delta}(t)$ are both observable stochastic processes.

Assumption 2.2. Decrements in $Q_{\text {sell }}^{\delta}(t)$ is the consequence of the execution of the market buy orders. ${ }^{6}$ Increments in $Q_{b u y}^{\delta}(t)$ is the consequence of the execution of the market sell orders.

Under the above assumption, we assume that the execution of market buy order of $j$ unit occur with rate $\lambda_{3}(j \mid X(t))$ but taking $P_{\text {sell }}(t)-p=\delta$. And similarly we assume that the execution of market sell order of $j$ unit occur with rate $\lambda_{1}(j \mid X(t))$ but taking $p-P_{\text {buy }}(t)=\delta$.

\section{$3 \quad$ Non-linear filtering}

Filtrations generated by observed limit orders are denoted by $\mathcal{G}_{t}=\sigma(Z(s): 0 \leq s \leq t)$, which is available to market participants. Global history is denoted by $\mathcal{F}_{t}=\mathcal{G}_{t} \vee \mathcal{F}_{t}^{X}$ where $\mathcal{F}_{t}^{X}=\sigma(X(s): 0 \leq s \leq t)$. We suppose that $X(t)$ is not directly observable for market participants and that the available information is specified by the filtration $\mathbb{G}=\left(\mathcal{G}_{t}\right)_{t>0}$ generated by the history of $Z(t)$.

Definition 3.1. Observation processes $Y_{k}(j ; t)$ counts the number of times that the order $k \in \mathcal{O}$ of size $j$ occurred during the time interval $[0, t]$.

Referring to the Assumption 2.1, precise expressions of the counting processes for six type of orders are given

\footnotetext{
${ }^{6}$ In order to eliminate the simultaneous events in our model formulation, we assumed this.
}

as follows.

$$
Y_{k}(j ; t)=N^{j}\left(\int_{0}^{t} \lambda_{k}(j, \ell(s-), X(s-)) d s\right), \quad k \in \mathcal{O}
$$

where $N^{j}(t)$ are mutually independent $\mathbb{P}$-Standard Poisson process which are independent of $X(t)^{7}$. We introduce an equivalent measure $\mathbb{Q}$ under which the processes $Y_{k}(j ; t), k \in \mathcal{O}$ are all standard Poisson. Let us define $L(t)$ as

$$
\begin{array}{r}
L(t)=1+\sum_{k=1}^{6} \sum_{j} \int_{0}^{t}\left[\lambda_{k}(j ; \ell(s-), X(s-))-1\right] \\
\times L(s-)\left(d Y_{k}(j ; s)-d s\right) .
\end{array}
$$

The solution of the above SDE can be represented by

$$
\begin{aligned}
L(t)=\prod_{k=1}^{6} \prod_{j} \exp & \left(\int_{0}^{t} \log \lambda_{k}(j ; \ell(s-), X(s-)) d Y_{k}(j ; s)\right. \\
& \left.-\int_{0}^{t}\left[\lambda_{k}(j ; \ell(s-), X(s-))-1\right] d s\right) .
\end{aligned}
$$

Thus we defined the new probability measure $\mathbb{Q}$ as $L(t)=\left.\frac{d \mathbb{P}}{d \mathbb{Q}}\right|_{t}$ and one sees that, under $\mathbb{Q}$, the counting processes $Y_{k}(j ; t), k \in \mathcal{O}$ are standard Poisson and furthermore we can choose $\mathbb{Q}$ so as to these six processes are independent of $X(t)$.

\subsection{Kushner-Stratonovich equation}

For an arbitrary bounded function $f$ on $\mathbb{R}^{2}$, let $\pi_{t}(f):=\mathbb{E}_{\mathbb{P}}\left[f(X(t)) \mid \mathcal{F}_{t}^{Y}\right]$ be the conditional expectation of $f(X(t))$ given $\mathcal{F}_{t}^{Y}:=\sigma\left(Y_{k}(j ; s): 0 \leq s \leq t, k \in\right.$ $\mathcal{O}, j \geq 1)$.

Theorem 3.2 (Kushner-Stratonovich equation). Let $\mathcal{A}$ be the infinitesimal generator of the process $X(t)$. Then $\pi_{t}(f)$ is the unique solution of the following SDE.

$$
\begin{aligned}
& \pi_{t}(f)= \pi_{0}(f)+\int_{0}^{t} \pi_{s}(\mathcal{A} f) d s \\
&+\sum_{k=1}^{6} \sum_{j} \int_{0}^{t}\left[\frac{\pi_{s-}\left(\lambda_{k}(j) f\right)}{\pi_{s-}\left(\lambda_{k}(j)\right)}-\pi_{s-}(f)\right] \\
& \quad \times\left(d Y_{k}(j ; s)-\pi_{s}\left(\lambda_{k}(j)\right) d s\right)
\end{aligned}
$$

Proof. The proof of the theorem follows in the same way as Zeng [10].

For the sake of numerical computation, KushnerStratonovich equation can be split into following two equations corresponding to whether the new order arrived or not at time $t$. Let $\tau_{n}$ be a random time that represents the $n$-th order appeared in the LOB.

\footnotetext{
${ }^{7}$ We assume that $\lambda_{k}, k=1,2, \cdots, 6$ are measurable functions.
} 
Between orders: $t \in\left(\tau_{n}, \tau_{n+1}\right)$

$$
\begin{aligned}
\pi_{t}(f) & =\pi_{\tau_{n}}(f)+\int_{\tau_{n}}^{t} \pi_{s}(\mathcal{A} f) d s \\
& -\sum_{j} \int_{\tau_{n}}^{t}\left[\pi_{s-}(\Lambda(j) f)+\pi_{s}(\Lambda(j)) \pi_{s-}(f)\right] d s
\end{aligned}
$$

where $\Lambda(j)=\sum_{k \in \mathcal{Q}} \lambda_{k}(j)$.

\section{At order: $t=\tau_{n}$ of type $k \in \mathcal{O}$}

$$
\pi_{\tau_{n}}(f)=\sum_{j} \frac{\pi_{\tau_{n}-}\left(\lambda_{k}(j) f\right)}{\pi_{\tau_{n}-}\left(\lambda_{k}(j)\right)} .
$$

In order to execute the particle filter algorithm practically, we discretize the time axis into small time interval $\Delta t=20$ milliseconds. Here, time stamps are denoted by $T_{1}, T_{2}, \cdots$. As in Kliemann et al. [6], Ceci and Gerardi [2] and Takada [7], it can be shown via the linearization method that the filter $\pi_{t}$ for $t \in\left[T_{m-1}, T_{m}\right)$ is expressed as

$$
\begin{aligned}
& \pi_{t}(f)=\frac{\int_{\mathbb{R}^{2}} \mathbb{E}_{T_{m-1}}^{\mathbb{Q}}\left[f\left(X_{t-T_{m-1}}\right) \Psi_{t-T_{m-1}}\right] \pi_{T_{m-1}}(d x)}{\int_{\mathbb{R}^{2}} \mathbb{E}_{T_{m-1}}^{\mathbb{Q}}\left[\Psi_{t-T_{m-1}}\right] \pi_{T_{m-1}}(d x)}, \\
& \Psi_{t-T_{m-1}}=\exp \left(-\int_{0}^{t-T_{m-1}} \Lambda\left(j ; X_{s}\right) d s\right)
\end{aligned}
$$

where $\pi_{T_{m-1}}(d x)$ is given below in (3). If the order of type $k \in \mathcal{O}$ renewed at $T_{m}$, we have

$$
=\frac{\int_{\mathbb{R}^{2}} \mathbb{E}_{T_{m-1}}^{\mathbb{Q}}\left[f\left(X_{T_{m}-T_{m-1}}\right) \Phi_{T_{m}-T_{m-1}}\right] \pi_{T_{m-1}}(d x)}{\int_{\mathbb{R}^{2}} \mathbb{E}_{T_{m-1}}^{\mathbb{Q}}\left[\Phi_{T_{m}-T_{m-1}}\right] \pi_{T_{m-1}}(d x)},
$$$$
\Phi_{T_{m}-T_{m-1}}=\lambda_{k}\left(j ; X_{T_{m}-T_{m-1}}\right) \Psi_{T_{m}-T_{m-1}} .
$$

\section{Enhancement via Support Vector Machine}

The above filtering model is designed to utilize the differences (changes for each $\Delta t$ ) of the state of the LOB. Since the initial state of the order book is not reflected, the filtering model can not utilize the exact current status of the LOB. However, in our numerical experience, Support Vector Machine (SVM), trained with the status of the LOB, possess great ability to predict future directions of the mid price. Inspired by this experience, we reconstruct the stochastic dynamics of the latent factor. More precisely, we assume that the trend function $a_{1}, a_{2}$ of $X(t)$ depend on the learning outcome of SVM trained with the current status of the
LOB. Let the shape of the LOB at time $T_{i}$ is identified with 10-dimensional vector

$$
\begin{aligned}
& \boldsymbol{z}_{i}=\left(Q_{\text {sell }}^{5 \delta}\left(T_{i}\right), Q_{\text {sell }}^{4 \delta}\left(T_{i}\right), \cdots, Q_{\text {sell }}^{\delta}\left(T_{i}\right),\right. \\
&\left.Q_{\text {buy }}^{\delta}\left(T_{i}\right), \cdots, Q_{\text {buy }}^{5 \delta}\left(T_{i}\right)\right)^{\top} .
\end{aligned}
$$

And then the history of the LOB for time interval $[0, t]$ is described as $Z_{[0, t]}=\left\{\boldsymbol{z}_{0}, \boldsymbol{z}_{1}, \cdots, \boldsymbol{z}_{\ell}\right\}$, with $T_{0}=0, T_{\ell}=t$.

Let $\pi\left(T_{i}\right)$ be a mid-price at time $T_{i}$ and define $I\left(T_{k}\right)=$ $\min \left\{j \mid \pi\left(T_{j+1}\right) \neq \pi\left(T_{j}\right), j \geq k\right\}$ as an index of the first mid-price movement. Training data for SVM is a set of sequence of prior trials $\left\{\boldsymbol{z}_{i} \in \mathbb{R}^{10}\right\}_{0<i<\ell}$ that have already been classified into two classes. In our case, classification means that each $\boldsymbol{z}_{i}$ has been assigned a label $y_{i} \in\{+1,-1\}$ depending on the direction of the first mid-price movement as follows.

$$
y_{i}= \begin{cases}+1 & \pi\left(T_{I\left(T_{i}\right)}\right)>\pi\left(T_{I\left(T_{i}\right)-1}\right) \\ -1 & \pi\left(T_{I\left(T_{i}\right)}\right)<\pi\left(T_{I\left(T_{i}\right)-1}\right)\end{cases}
$$

Trained SVM finds maximum margin hyper plane in a feature space as a final decision boundary. The resulting decision boundary is defined by

$$
h(\boldsymbol{z})=\operatorname{sign}\left(\sum_{i=0}^{\ell} y_{i} \alpha_{i} K\left(\boldsymbol{z}, \boldsymbol{z}_{i}\right)+b\right),
$$

where $K$ is called kernel function. Quadratic Programing problem below is solved to first find the value of parameters $\alpha_{i}$. Parameter $C$ controls the trade-off between margin and misclassification error.

$$
\begin{array}{ll}
\max _{\boldsymbol{\alpha}} & \sum_{i=0}^{\ell} \alpha_{i}-\frac{1}{2} \sum_{i, j} \alpha_{i} \alpha_{j} y_{i} y_{j} K\left(\boldsymbol{x}_{i}, \boldsymbol{x}_{j}\right) \\
\text { s.t. } & 0 \leq \alpha_{i} \leq C, \quad \sum_{i=0}^{\ell} \alpha_{i} y_{i}=0 .
\end{array}
$$

We select $K$ as a Gaussian kernel defined as $K\left(\boldsymbol{z}_{i}, \boldsymbol{z}_{j}\right)=$ $\exp \left(-\left\|\boldsymbol{z}_{i}-\boldsymbol{z}_{j}\right\|^{2} / \sigma^{2}\right)$ and then the trained SVM will return predicted direction, called a label $y_{\ell+1}=$ $h\left(\boldsymbol{z}_{\ell+1}\right) \in\{+1,-1\}$ for new trial data $\boldsymbol{z}_{\ell+1}$.

The above set of procedures can be used to define the trend function $a_{1}$ and $a_{2}$ so that $y=+1(y=-1)$ induces downward trend for $X_{1}\left(X_{2}\right)$ and upward trend for $X_{2}\left(X_{1}\right)$. For numerical implementation, we assume that $d B_{1}$ and $d B_{2}$ are independent and

$$
\begin{aligned}
a_{i}\left(t, X_{i}(t), Z_{[0, t]}\right) & =\bar{q}_{i} \cdot\left(\bar{a}_{i} \cdot y_{t}-X_{i}(t)\right), \\
b_{i}\left(t, X_{i}(t), Z_{[0, t]}\right) & =\bar{b}_{i}, \quad \bar{q}_{i}, \bar{a}_{i}, \bar{b}_{i} \in \mathbb{R} .
\end{aligned}
$$

Therefore $X_{i}(t)$ obeys regime switching OrnsteinUhlenbeck process, i.e., $X_{i}(t)$ has high reverting revel $\bar{a}_{i}$ if the trained SVM returned $y=+1$, and has low reverting revel $-\bar{a}_{i}$ if the SVM returned $y=-1$. 


\section{Numerical Algorithm via Particle Filter}

It is necessary to approximate the expected values appeared in equations (1) and (2) so as to numerically compute the filter $\pi_{t}(f)$. In practice, we take $f$ as an identity map and employ branching particle filter to approximate the conditional distribution $\mathbb{P}\left(X_{t} \in \bullet \mid \mathcal{G}_{t}\right)$ as in Del Moral and Miclo [4]. Particle filter is a method to approximate the conditional distribution $\mathbb{P}\left(X_{t} \in \bullet \mid \mathcal{G}_{t}\right)$ with some suitable discrete random measures of the form $\mathbb{P}\left(X_{t} \in \bullet \mid \mathcal{G}_{t}\right) \approx \sum_{p} \eta_{t}^{p} \delta_{x_{t}^{p}}(\bullet)$ with some sample points $\left\{x_{t}^{p}\right\}$ and their consistent stochastic weights $\left\{\eta_{t}^{p}\right\}$. The algorithm is constructed with the sequence of occupation measures $\left\{\tilde{\pi}_{T_{i}}\right\}_{i=1,2, \cdots}$ approximating the conditional distributions $\pi_{X_{T_{i}} \mid \mathcal{G}_{T_{i}}}:=\mathbb{P}\left(X_{T_{i}} \in \bullet \mid \mathcal{G}_{T_{i}}\right)$ for each time step.

Let $\boldsymbol{x}_{i}=\left(x_{i}^{1}, x_{i}^{2}, \cdots, x_{i}^{n_{i}}\right)$ denote the set of $n_{i}$ particles at time $T_{i}$ living in the state space of $\boldsymbol{X}=\left(X_{1}, X_{2}\right)$. We modify the prediction stage of the algorithm proposed by Takada [7] to include the learning outcome by SVM.

Algorithm 5.1. In order to derive the discrete filter distribution $\left\{\tilde{\pi}_{T_{i}}\right\}_{i=0,1, \ldots}$, proceed the following;

\section{Step 1. Initial discrete distribution}

The initial discrete distribution $\tilde{\pi}_{0}$ is given by the occupation measure of $n_{0}$ particles of mass $1 / n_{0}$, that is,

$$
\tilde{\pi}_{0}=\frac{1}{n_{0}} \sum_{k=1}^{n_{0}} \delta_{x^{k}(0)} .
$$

Here $\boldsymbol{x}_{0}=\left(x^{1}(0), x^{2}(0), \cdots, x^{n_{0}}(0)\right)$ represents independent draws from the initial distribution $\pi_{0}:=$ $\mathbb{P}\left(X_{0} \in \bullet\right)$.

Step 2. Prediction stage combined with $S V M$ Given the particles $\boldsymbol{x}_{i}=\left(x_{i}^{1}, \cdots, x_{i}^{n_{i}}\right)$ at $T_{i}$, generate $n_{i}$ independent trajectories

$$
\left(\boldsymbol{X}_{s}\right)_{T_{i} \leq s \leq T_{i+1}}=\left(\left(X_{s}^{1}\right)_{T_{i} \leq s \leq T_{i+1}}, \cdots,\left(X_{s}^{n_{i}}\right)_{T_{i} \leq s \leq T_{i+1}}\right)
$$

such that, for each $p \in\left\{1, \cdots, n_{i}\right\}, \boldsymbol{X}_{s}^{p}$ starts from $x_{i}^{p}$ at time $T_{i}$ and then follows the $S D E$ of $\boldsymbol{X}=\left(X_{1}, X_{2}\right)$ of the form

$$
d X_{i}(t)=\bar{q}_{i} \cdot\left(\bar{a}_{i} \cdot y_{t}-X_{i}(t)\right) d t+\bar{b}_{i} \cdot d B_{i}(t) .
$$

where $y_{t}$ is the predicted outcome of the trained SVM.

Step 3. Updating stage

Compute

$$
\tilde{w}_{i+1}^{p}=\exp \left(-\int_{T_{i}}^{T_{i+1}} \sum_{j} \Lambda\left(j ; \boldsymbol{X}_{s}^{p}\right) d s\right)
$$

for each $\boldsymbol{X}_{s}^{p}$ obtained in the last prediction stage. We redefine $w_{i+1}^{p}$ depending on whether the new order arrived during $\left(T_{i}, T_{i+1}\right]$ or not. That is, if there is no
$L O B$ renewals during $\left(T_{i}, T_{i+1}\right]$, set $w_{i+1}^{p}:=\tilde{w}_{i+1}^{p}$, and if an order $k \in \mathcal{O}$ of $j$ units arrived at $T_{i+1}$, set $w_{i+1}^{p}:=\lambda_{k}\left(j ; \boldsymbol{X}_{T_{i+1}}^{p}\right) \times \tilde{w}_{i+1}^{p}$. Compute

$$
v_{i+1}^{p}=\frac{n_{i} w_{i+1}^{p}}{\sum_{q=1}^{n_{i}} w_{i+1}^{q}}
$$

for every $p=1, \cdots, n_{i}$ and obtain $\left\{o_{i+1}^{p}\right\}_{p=1, \cdots, n_{i}}$

$o_{i+1}^{p}= \begin{cases}{\left[v_{i+1}^{p}\right]} & \text { with probability } 1+\left[v_{i+1}^{p}\right]-v_{i+1}^{p} \\ {\left[v_{i+1}^{p}\right]+1} & \text { with probability } v_{i+1}^{p}-\left[v_{i+1}^{p}\right]\end{cases}$

where $[v]$ stands for the integer part of $v$. Denote by $n_{i+1}=\sum_{p=1}^{n_{i}} o_{i+1}^{p}$ the total number of particles at $T_{i+1}$. Then each particle $x_{i}^{p}$ at $T_{i}$ independently generates $o_{i+1}^{p}$ offsprings of $\left(\boldsymbol{X}_{s}^{p}\right)_{T_{i} \leq s \leq T_{i+1}}$ starting at $x_{i}^{p}$ and denoted by $\boldsymbol{x}_{i+1}=\left(x_{i+1}^{1}, \cdots, x_{i+1}^{n_{i+1}}\right)$.

Thus one can update the approximated distribution

$$
\tilde{\pi}_{t_{k+1}}=\frac{1}{n_{k+1}} \sum_{p=1}^{n_{k+1}} \delta_{x_{k+1}^{p}}
$$

Step 4. Proceed from $i$ to $i+1$ and go to Step 2 until some time horizon.

In Step 3, each particle is replaced by the particles of which the number is randomly given by $o^{p}$. This procedure is worked in a consistent manner; particles with small weights $w^{p}$ have almost zero offspring while those with large weights are replaced by several offspring.

While at the same time, computational time of the above particle filter algorithm is crucial for practical use. In our numerical analysis, we took the minimum unit as $\Delta t=20$ milliseconds and set $n_{0}=5000$ for initial number of particles. As a result, the calculation time for the time interval $\Delta t$ required about 10 milliseconds at most with Core i7 $3.5 \mathrm{GHz}$ CPU. This would be changed as a function of $n_{0}$ almost linearly, which would help us to choose the best suited number of initial particles.

\section{$6 \quad$ Numerical results}

Figure 3 and 4 illustrates the transitions of the percentile points of the occupation measure $\tilde{\pi}_{t}$ of $X_{1}(t)$ and $X_{2}(t)$ respectively, calculated every 1 second from 9:00 am, opening time ${ }^{8}$, to 9:30 am as of 1st June 2012 in Osaka stock market in Japan ${ }^{9}$. Here we choose the model parameters listed in Table 1.

The solid black line indicate the history of the mid price with right axis. Roughly, when mid price move to downward, $X_{1}$ tends to jump up and $X_{2}$ tends to jump down as expected. However, these are not always true because $X(t)=\left(X_{1}(t), X_{2}(t)\right)$ is sensitive to overall dynamics of the LOB.

\footnotetext{
${ }^{8}$ More precisely, we exclude the data for first few seconds because the best ask and best bid prices have not yet split.

${ }^{9}$ These tick data were available from Rakuten securities Co., Ltd. in Japan.
} 
Table 1: Parameter set

\begin{tabular}{ccccccccc}
$g_{0}$ & $g_{1}$ & $h_{0}$ & $h_{1}$ & $u_{0}$ & $u_{1}$ & $v_{0}$ & $v_{1}$ & \\
\hline 0.05 & 0.5 & 0.05 & 0.5 & 0.05 & 0.5 & 0.05 & 0.5 & \\
& & & & & & $\bar{b}_{1}$ & $\bar{b}_{2}$ & $\rho$ \\
$\alpha$ & $\beta$ & $\gamma$ & $\kappa$ & $q_{1}$ & $q_{2}$ & & \\
\hline 1.8 & 0.5 & 0.5 & 0.5 & 0.2 & 0.2 & 0.14 & 0.14 & 0
\end{tabular}

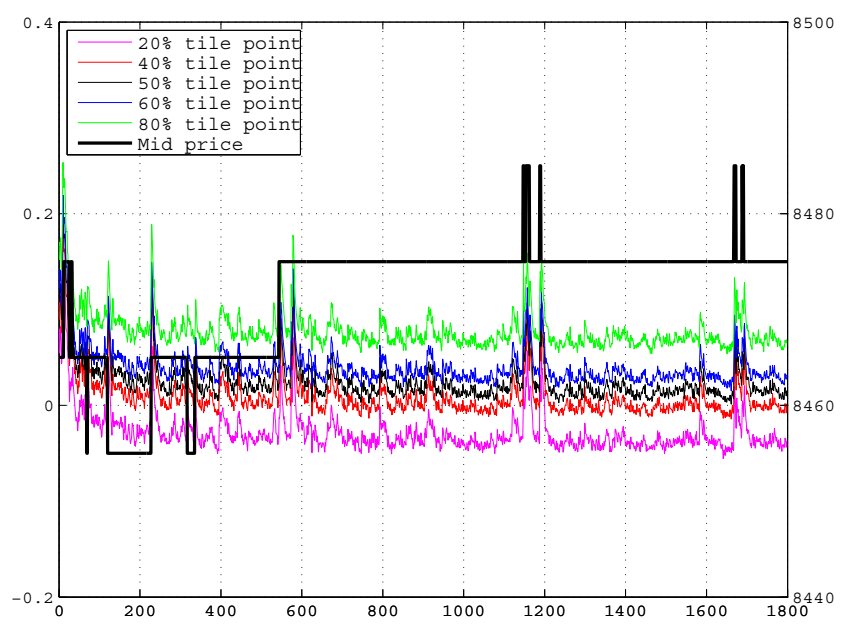

Fig. 3: Transitions of the filter $\tilde{\pi}_{t}$ of $X_{1}(t)$ calculated for intraday data as of 1st June 2012

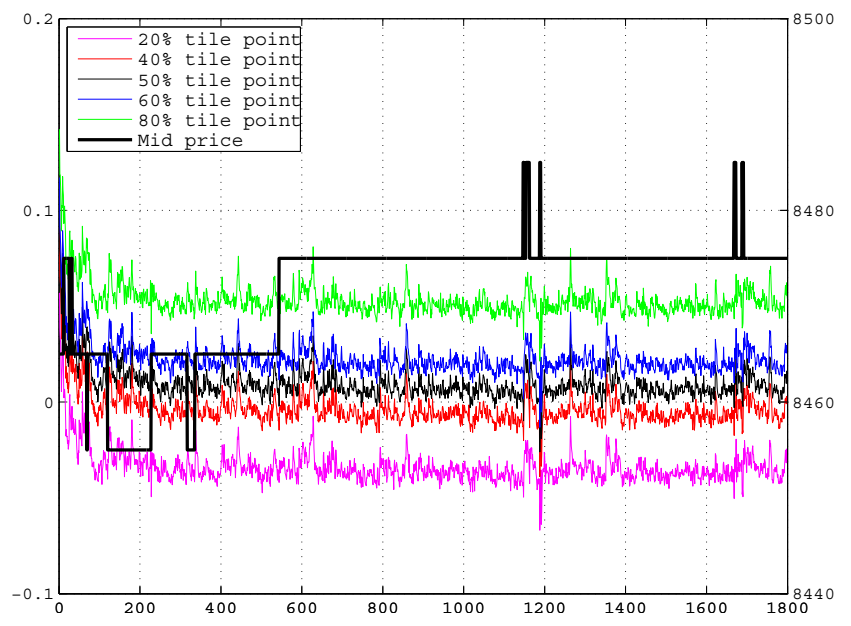

Fig. 4: Transitions of the filter $\tilde{\pi}_{t}$ of $X_{2}(t)$ calculated for intraday data as of 1st June 2012

Updated set of $\left(X_{1}(t), X_{2}(t)\right)$ enable one to estimate the degree of market activities and then the arrival rates of future market orders would be evaluated. Probability of the mid price move would be represented by the intensities as follows. Intensity that the mid price goes up at time $t$, given information until time $t-=t-\Delta t$, is obtained by

$$
\begin{aligned}
\sum_{j>Q_{\text {sell }}^{\delta}(t-)} & \lambda_{3}(j, X(t-)) \\
= & \sum_{j>Q_{\text {sell }}^{\delta}(t-)} \bar{\lambda}_{3}(10, X(t-)) \times J_{3}(j, X(t-)) .
\end{aligned}
$$

And the intensity that the mid price goes down at time $t$, given information until time $t-=t-\Delta t$, is obtained by

$$
\begin{aligned}
\sum_{j>Q_{\text {buy }}^{\delta}(t-)} \lambda_{1}(j, X(t-)) & \\
= & \sum_{j>Q_{b u y}^{\delta}(t-)} \bar{\lambda}_{1}(10, X(t-)) \times J_{1}(j, X(t-)) .
\end{aligned}
$$

The authors would investigate the validity of the above quantities as a further research.

Acknowledgements The authors are grateful to Yasuo Suzuki for technical support especially for tick data handling and also grateful to participants of the 47th ISCIE International Symposium on Stochastic Systems Theory and Its Applications (SSS'15) for fruitful discussions. We thank two anonymous reviewers for their careful reading and valuable comments, which have contributed to the present version.

\section{References}

[1] Bouchaud, J.-P., M., Mezard. and M. Potters, 2002, "Statistical properties of stock books: Empirical results and models", Quantitative Finance, 2, 251-256.

[2] Ceci, C. and A. Gerardi, 2006, "A Model for High Frequency Data under Partial Information: A Filtering Approach", International Journal of Theoretical and Applied Finance, 9(4), 555-576.

[3] Cont, R., Stoikov, S. and Talreja, R, 2010, "A Stochastic Model for Order Book Dynamics" Operations Research, 58, No. 3, 549-563.

[4] Del Moral, P. and L. Miclo, 2000, "Branching and Interacting Particle Systems. Approximations of Feynman-Kac Formulae with Applications to Nonlinear Filtering", Seminaire de probabilities (Strasbourg), tome 34, 1-145.

[5] Huang, He and A. H. Kercheval, 2012, "A Generalized Birth-Death Stochastic Model for HighFrequency Order Book Dynamics", Quantitative Finance, 12 (4), 547-557.

[6] Kliemann, W. H., G. Kock, and F. Marchetti, 1990, "On the Unnormalized Solution of the Filtering Problem with Counting Process Observations", IEEE Transactions and Information Theory, 36(6), 1415 - 1425. 
[7] Takada, H., 2015, "Filtering Model for Order Book Dynamics", Research Institute for Mathematical Sciences Kokyuroku, 1933, 70 - 88.

[8] Xiong, J. and Y. Zeng, 2011, "A Branching Particle Approximation to a Filtering Micromovement Model of Asset Price", Statistical Inference for Stochastic Processes, 14, 111-140.

[9] Zovko, I. and J. D. Farmer, 2002, "The power of patience; A behavioral regularity in limit order placement", Quantitative Finance, 2, 387-392.

[10] Zeng, Y., 2003, "A Partially-Observed Model for Micro-movement of Asset Prices with Bayes Estimation via Filtering", Mathematical Finance, 13, 411-444. 